\title{
Perioperative glucose management in patients undergoing cardiac surgery
}

Hemeryck M., Verburgh P., Vandewiele K., Bouchez S., Wouters P., De Hert S.

University Hospital Ghent, Dept of Anaesthesiology, Ghent, Belgium

\section{Background}

Despite the development of various insulin protocols, perioperative hypo- and hyperglycaemic events during cardiopulmonary bypass remain a point of concern.

The University of Ghent Insulin Protocol has been found to keep perioperative glycaemia within narrow limits, while diabetes mellitus, use of corticosteroids and age are risk factors for deviation of pre-induction glycaemia.

\section{Goal of Study}

In this study we sought to identify independent risk factors for significant perioperative hyperglycaemic (> $180 \mathrm{mg} / \mathrm{dL}$ ) and hypoglycaemic ( $<70 \mathrm{mg} / \mathrm{dL}$ ) events when using the UGIP.

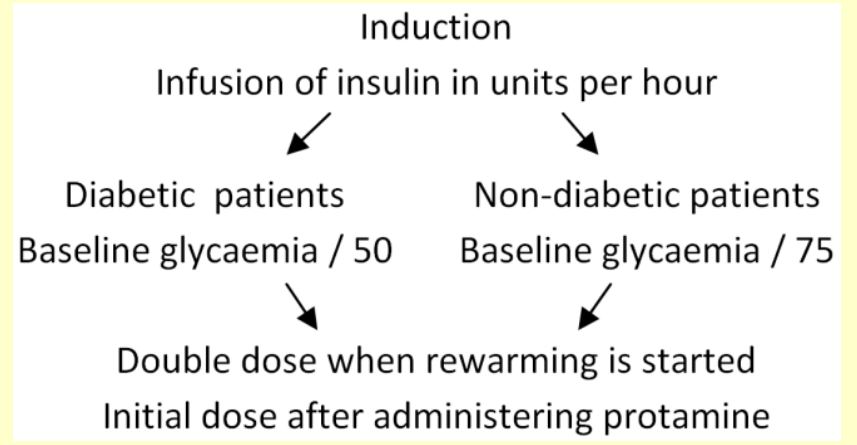

\section{Methods}

Retrospective cohort study.

After exclusion, data from 776 cardiac surgery patients remained.

Relative risks were calculated for the following variables:

- Redo operation

- CPB duration > $60 \mathrm{~min}$

- Age $>70$ years

- Gender

- Temperature on $\mathrm{CPB}<32^{\circ} \mathrm{C}$

- Nadir haematocrit < 24\%

- Inotropic and/or vasoactive support

- Corticosteroids

- Diabetes mellitus

The statistically significant variables were included in a multiple regression analysis.

\section{$\underline{\text { Results }}$}

\section{Backward Stepwise Regression}

$\mathrm{RR}$
Multiple linear regression

\begin{tabular}{llcccc}
\hline & & $\mathrm{RR}$ & $95 \% \mathrm{Cl}$ & $\mathrm{p}$ value & $\mathrm{p}$ value \\
\hline Hyperglycaemia & Male gender & 0.34 & $0.13-0.90$ & 0.029 & 0.120 \\
\cline { 2 - 6 }$(\mathrm{n}=16,2 \%)$ & Inotropic support & 3.81 & $1.40-10.37$ & 0.01 & 0.019 \\
\cline { 2 - 6 } & Corticosteroids & 5.83 & $1.96-17.30$ & 0.008 & 0.006 \\
\hline Hypoglycaemia & Inotropic support & 4.87 & $1.40-17.09$ & 0.035 & 0.536 \\
\cline { 2 - 6 }$(\mathrm{n}=13,1.7 \%)$ & Diabetes mellitus & 4.87 & $1.23-11.13$ & 0.028 & 0.013 \\
\hline
\end{tabular}

\section{Conclusion}

The following variables appeared to be independent risk factors for hyper- or hypoglycaemic events when using the UGIP:

- Glycaemia < $70 \mathrm{mg} / \mathrm{dL}$ : corticosteroids and inotropic and/or vasoactive agents.

- Glycaemia > 180 mg/dL: diabetes mellitus. 